\title{
Randomized Trial of Bulb Syringes for Ear- wax: Impact on Health Service Utilization
}

\author{
Richard Coppin, BMed Sci, BM, BS, \\ DRCOG $^{1}$ \\ Dorotby Wicke, $\mathrm{RN}^{1}$ \\ Paul Little, FRCGP, MRCP, MD, BA, \\ $M B B S^{2}$
}

'Research \& Development Office, The Surgery, Station Road, Overton, Hampshire,

United Kingdom

${ }^{2}$ University of Southampton Primary Medical Care, Aldermoor Health Centre, Aldermoor Close, Southampton, United Kingdom
Conflicts of interest: authors report none.

\section{CORRESPONDING AUTHOR}

Richard Coppin, BMed Sci, BM, BS DRCOG

Research \& Development Office

The Surgery

Station Road

Overton, Hants, UK RG25 3DU

richard.coppin@nhs.net

\begin{abstract}
PURPOSE Bulb syringes can be used for the self-clearance of earwax and, in the short term, appear effective. We compared the long-term effectiveness of selfirrigation using a bulb syringe with routine care in United Kingdom (UK) family practice clinics where irrigating ears to remove wax is a common procedure.
\end{abstract}

METHODS We assessed the impact on health service utilization as a follow-up to a single-blind, randomized, controlled trial of 237 patients attending 7 UK family practice clinics with symptomatic, occluding earwax who were randomized to an intervention group (ear drops, bulb syringe, instructions on its use and re-use) or a control group (ear drops, then clinic irrigation). After 2 years, a retrospective notes search for earwax-related consultations was carried out. We used an intention-totreat analysis to assess differences in dichotomous outcomes between groups.

RESULTS In the 2-year trial follow-up, more control group patients returned with episodes of earwax: 85 of $117(73 \%)$ control vs 70 of $117(60 \%)$ intervention, $\chi^{2}=4.30 ; P=.038$; risk ratio $1.21(95 \% \mathrm{Cl}, 1.01-1.37)$. The numbers of consultations amounted to 1.15 (control) vs 0.64 (intervention) (incidence rate ratio 1.79; $95 \% \mathrm{Cl}, 1.05-3.04, P=.032)$, ie, a difference of 0.50 consultations, thus saving a consultation on average for every 2 people.

CONCLUSION For patients who have not already tried bulb syringes, self-irrigation using a bulb syringe significantly reduces subsequent demand for ear irrigation by health professionals. Advocating the initial use of bulb syringes could reduce demand for ear irrigation in family practice clinics.

Ann Fam Med 2011;9:110-114. doi:10.1370/afm.1229.

\section{INTRODUCTION}

lthough there are few data, it has been estimated that each year 12
million people in the United States seek medical care for problems
with earwax, and 8 million ear irrigation procedures are carried out. ${ }^{1}$ To remove symptomatic wax, current professional guidelines advocate the use of ear drops to soften wax, followed, if necessary, by irrigation by a trained clinician. ${ }^{2}$ In UK family practice, although ear irrigation is now carried out more often by family practice nurses than by physicians, ${ }^{3}$ it still represents a significant health cost. ${ }^{4}$ Drops are frequently used to soften wax before irrigation, but there is limited evidence to suggest that self-treatment with drops alone is a satisfactory treatment without some form of mechanical removal of wax. ${ }^{5}$ In the United States and many other countries, but not the United Kingdom, soft plastic bulb syringes are widely available over the counter or via the Internet and can be used to self-irrigate ears to clear wax. Although such home irrigation has received professional endorsement, ${ }^{6}$ little is known about its effectiveness. A small study in Ireland suggested that bulb syringes were effective in removing previously softened wax. ${ }^{7}$ In a randomized trial we showed a significant reduction in self-reported symptoms of earwax after using bulb syringes, which achieved approximately two-thirds of the improvement achieved by conventional irrigation, and $75 \%$ of those using a bulb syringe would use it again. ${ }^{8}$ We also reported that our study found no evidence of harm after 
self-use of bulb syringes. As a longer term follow-up of that trial, we now report the impact of offering patients bulb syringes on the subsequent demand for consultations for earwax and for irrigation of ears carried out by primary care clinicians.

\section{METHODS}

Ethical approval was granted by the UK North and Mid Hampshire Local Research Ethics Committee (Reference 03/A/057). Two hundred thirty-seven adult patients, with symptoms of blockage and visible, occluding wax, were recruited from 7 family practice clinics in the south of England in 2004. A clinic nurse handed participants a numbered, sealed envelope, which had previously been randomized using random number tables by a member of the research team not involved in recruitment or data collection. The envelope handed to intervention patients contained ear drops, a 25 -mL bulb syringe, and written instructions describing its use, which also stated that the bulb syringe could be cleaned and reused. The envelope for control patients was of identical weight, size, and texture, and it contained ear drops, a roll of card of identical weight to a bulb syringe, and instructions on usual treatment, which normally comprised ear irrigation carried out by a clinic nurse after use of ear drops. A further group of patients ( $n=128)$, while not consenting to randomization, did agree to their notes being searched. At 2 weeks after randomization, all patients were reassessed for blockage by the clinic nurse who cleared by irrigation those patients who ears had persistent occluding wax. ${ }^{8}$ In August 2007 one of the research team (D.W.) who was blinded to allocation reviewed the records for the 2-year period from 6 weeks after randomization for consultations for further problems with earwax or its treatment and for related hospital referrals.

To estimate the burden of ear care in the general population, we asked the clinic nurses to check the written and computerized records of the study population for ear irrigation consultations in the 5 years before randomization. We also asked the clinics to undertake a retrospective search of the computerized records of their entire clinic population for all patients attending for ear irrigation procedures in the year before the study. In the United Kingdom, patients are registered with a local National Health Service (NHS) family practice clinic that is responsible for maintaining their lifelong, personal health record, which includes all primary and secondary care contacts. In most UK family practice clinics, patient records are now held on computerized systems into which staff routinely input data, including such procedures as ear irrigation, using a common system of coding. Searching structured fields for particular codes is routinely carried out for audit and other purposes.

\section{Sample Size}

We did not do an a priori sample size calculation for the follow-up data: the original power calculation ${ }^{8}$ was based on the assumption of a $15 \%$ difference in wax clearance (75\% vs $90 \%)$, which required 100 patients per group plus $15 \%$ for loss to follow-up (for $\alpha=.05$ and $\beta=.2$ ); we assumed the follow-up study has power to detect a similar $15 \%$ difference in repeat consultations and more power to assess repeated consultation rates as a continuous outcome.

\section{Analysis}

We used an intention-to-treat analysis and the $\chi^{2}$ test and logistic regression to assess differences in dichotomous outcomes between groups (eg, return with episodes of earwax), and negative binomial regression for the repeat consultation rate data. Patients commonly returned with further episodes of occluding earwax in the follow-up period, and because odds ratios can exaggerate the apparent benefit for common problems, we converted the odds ratio to risk ratio using the method of Zhang and Yu. ${ }^{9}$

\section{RESULTS}

Of the 434 participants eligible for the study, 118 were allocated to the intervention group, and 119 were allocated to the control group. Data from 117 participants in each group were available for analysis after 2 years (Figure 1 displays the participant flowchart). The intervention group was $66 \%$ male with a mean age of 57 years ( $\mathrm{SD}=14$ years), whereas the control group was $63 \%$ male with a mean age of 55 years ( $S D=16$ years).

Patients in both groups were very well balanced at baseline (comparing bulb syringe vs control groups, respectively, mean symptoms 2.37 vs 2.41 ; right ear completely blocked $63 \%$ vs $62 \%$; left ear completely blocked $67 \%$ vs 69\%). ${ }^{8}$ In the control group (clinic irrigation) more patients returned with episodes of earwax in the 2-year follow-up (irrigation, advice, or other consultations): 85 of 117 (73\%) control vs 70 of $117(60 \%)$ in the intervention ( bulb syringe) group $\left(\chi^{2}=4.30\right.$; $P=.038$; risk ratio $=1.21 ; 95 \% \mathrm{CI}, 1.01-1.37)$.

In the 2-year follow-up period, the mean number of consultations for earwax amounted to 1.15 (control) vs 0.64 ( bulb syringe), ie, a difference of 0.50 consultations, and an estimated $79 \%$ higher consultation rate for the control group (incidence rate ratio $=1.79 ; 95 \%$ $\mathrm{CI}, 1.05-3.04 ; P=.032)$, saving 1 consultation on average for every 2 patients. There was a significant differ- 
ence in irrigation procedures of 0.91 (control) vs 0.46 ( bulb syringe), a difference of 0.44 consultations, an estimated $96 \%$ higher clinic irrigation procedure rate in the control group (incidence rate ratio $=1.96 ; 95 \%$ CI, $\left.1.15-3.36_{;} P=.014\right)$.

During the period from randomization (ie, the 6 -week study period and the subsequent 2 years), the mean number of irrigation procedures was 1.92 and 0.74 in the control and bulb syringe groups, respectively. In those randomized to bulb syringe, 49 of 99 (49\%) had no record of attendance for irrigation during either the initial study period or in the subsequent 2 years.

There were fewer adverse events related to irrigation in the bulb syringe group 2 of $117(1.7 \%)$ vs 5 of 117 (4.2\%) in the control group, but this difference was not significant (Fishers exact test, $P=.446$ ), and there was 1 referral to an ear, nose, and throat specialist in the control group and none in the bulb syringe group. Prescriptions related to earwax were few in both groups (mean $=0.05$ control vs 0.09 bulb syringe) and there were similar earwax symptom severity scores ${ }^{8}$ (control $1.91, \mathrm{SD}=0.98$, vs bulb syringe $1.85, \mathrm{SD}=0.88$ ).

In the 5 years before recruitment, of 362 patients in the study population whose records were searched (70 were not available), 223 (62\%) had a record of clinic irrigation equivalent to $12 \%$ per annum. There were 644 irrigation procedures carried out on the 362 patients (mean $=1.78, \mathrm{SD}=2.16$ ).

Figure 1. Participant flowchart.

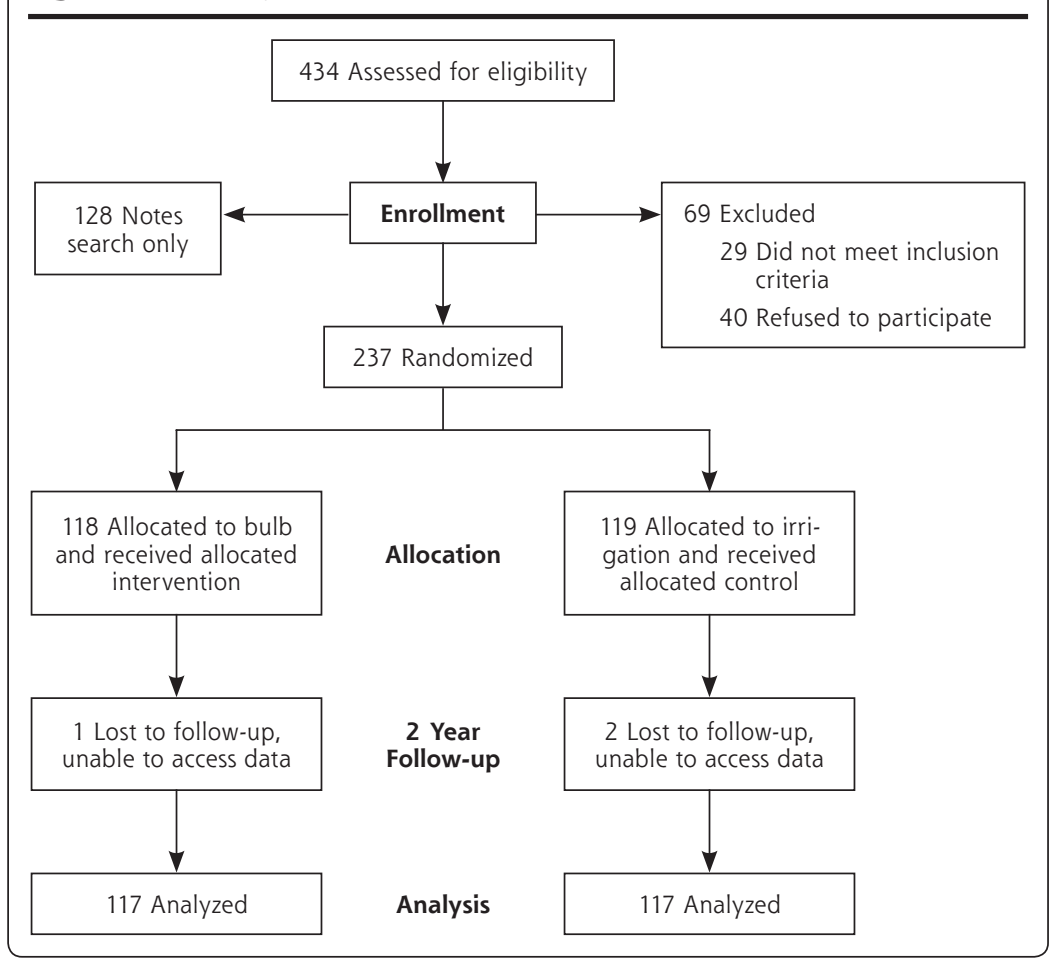

The search of the computerized records of all patients in the 7 study family practice clinics was limited by the capability of the different computer systems and how data were recorded in each clinic. Of the total of 72,261 patients registered in 6 of the participating clinics, 1,819 patients $(2.52 \%)$ had their ears irrigated in the year before the study. There were 5 participating clinics for which the number of procedures was available, and data from these 5 clinics, with 63,535 registered patients, showed that the number of irrigation procedures was 1,955 , equivalent to $3.08 \%$ of patients registered. We report findings for both the number of patients and the number of procedures, because our figures are more robust than previously published estimates.

\section{DISCUSSION}

\section{Summary of Main Findings}

Of patients seeking relief of ears blocked with wax, which was subsequently cleared, those originally given a bulb syringe for self-use had about one-half the rate of irrigation consultations of those treated by nurse irrigation in the subsequent 2 years. This rate is similar to our finding that about one-half of all those given a bulb syringe at randomization had not required clinic irrigation more than 2 years later. The retrospective audit found that each year about $2.5 \%$ of the population attends family practice clinics to have their ears irrigated and that the number of irrigation procedures equates to $3.1 \%$ of the population.

\section{Strengths and Limitations of the Study}

As we did not seek data on the further use of the bulb syringes issued, we assume that the reduction in attendance and clinic irrigation rates during the 2-year follow-up was due to patients reusing the devices. The instructions issued with the bulb syringe indicated that the device could be cleaned and reused but patients were not otherwise encouraged in their index consultation to manage themselves in future episodes. Had we done so, it is possible that we might have increased the effectiveness of self-care with bulb syringes. Similarly we did not enquire whether control patients were obtaining bulb syringes elsewhere, so we possibly underesti- 
mated the effect size of the intervention. Given the general difficulty in obtaining bulb syringes in the United Kingdom at that time, it seems unlikely patients were able to obtain them.

There are some difficulties in extrapolating our findings to the United States, where bulb syringes are more widely available than in the United Kingdom, and some patients may already have tried using a bulb syringe before seeking help. For those who have not tried a bulb syringe, however, our findings should still apply.

Our data apply only to a 2 -year follow-up period, and it is possible that the use of bulb syringes only defers the need for clinic irrigation. It is equally possible, however, that patients' ability to treat symptoms at an earlier stage than might justify a clinic appointment, and to irrigate their ears more frequently, might tend to reduce the need for intervention by clinicians.

Our retrospective audit data were limited by the capability and usage of the different computer systems. Although it is possible that some patients might have been treated elsewhere, resulting in our data being incomplete, it is unlikely. In the United Kingdom there is very little availability of ear care services other than those provided by the NHS, and the family practice clinic where the patient is registered is invariably notified when the patient attends a hospital or other clinics. As we studied a small sample of family practice clinics, it is possible that the study clinics may not be representative of the United Kingdom or elsewhere.

Our finding of a marked difference in rates of clinic irrigation between the study population and the general population ( $12 \%$ vs $2.5 \%$ ) is not surprising. Those attending with earwax problems presumably represent a minority population characterized by greater use of ear care services and associated factors, particularly age.

\section{Comparison With Existing Literature}

The reduction in health service utilization in our intervention group is consistent with our previous report that showed a reduction in symptoms of blockage following use of a bulb syringe and an observed clearance of wax in around $50 \%$ of patients (although nurse irrigation achieved clearance in $70 \%) .{ }^{8}$ In a small study Harris found an observed clearance rate of $57 \%$ after use of a bulb syringe in those with completely obscured eardrums previously treated with ear drops. ${ }^{7}$

Our retrospective audit found that $2.5 \%$ of the population attend primary care clinics for ear irrigation annually, compared with previous estimates of $3.9 \%$ for the United Kingdom, ${ }^{10}$ and 2.8\% for the United States. ${ }^{11}$ Our finding that the number of irrigation procedures equates to $3.1 \%$ of the population differs from the $7 \%$ found in a Scottish primary care questionnaire survey, which estimated the number of ears rather than patients. ${ }^{12}$ Our figures are based on a retrospective search of patient records, however, and relate to records of procedures carried out. As such, they are probably a more reliable figure on which to estimate overall workload and utilization of health care resources.

\section{Safety}

Although irrigation in general can result in perforation of the tympanic membrane, ${ }^{13,14}$ the data refer to the use of manual syringes or oral jet irrigators that operate at higher pressures than current ear irrigators and do not refer to bulb syringes. A recent systematic review looking specifically at adverse events commented on the limited data but concluded that irrigation with bulb syringes appeared to be reasonably safe. ${ }^{4}$ We previously reported that a search of the US Government adverse-event register showed no events ascribed to the use of bulb syringes. ${ }^{8}$ The apparent safety of this procedure may be explained by our personal experience that self-irrigation with a of bulb syringe enables fine adjustment of the pressure of the water to avoid discomfort and presumably damage.

\section{Implications for Future Research or Clinical Practice}

These findings support the tentative suggestion of Clegg and colleagues ${ }^{4}$ that, rather than routine attendance with a clinician, self-treatment with drops and then self-irrigation may offer a significantly less costly alternative, with patients attending family practice clinics only if they are unable to clear the problem satisfactorily. Larger effectiveness studies, as well as research to assess the incidence of adverse events, are required to inform future policy.

Extrapolating from these data, clinic staff in the United Kingdom carry out nearly 2 million irrigation procedures per year. This equates to a US figure of nearly 10 million per year. Encouraging the initial use of bulb syringes could, certainly in the subsequent 2 years, potentially reduce the number of clinic irrigations by around 0.9 million in the United Kingdom. It is likely that the potential for such a reduction in the United States is less, as bulb syringes are already available. In both countries such a policy might help to demedicalize the problem of earwax removal, saving patients the time and cost involved in clinic attendance.

To read or post commentaries in response to this article, see it online at http://www.annfammed.org/cgi/content/full/9/2/110.

Key words: Cerumen; adult; ear canal; family practice; therapeutic irrigation; medical records

Submitted May 9, 2010; submitted, revised, September 29, 2010; accepted October 25, 2010. 
Findings from this study were given as an oral presentation at the UK Society for Academic Primary Care Annual Scientific Meeting, St Andrews University, July 9, 2009, Scotland.

Funding support: The study was funded by the UK Royal College of General Practitioners Scientific Foundation Board, reference SFB/2003/32, and supported by UK Department of Health Support for Science funding.

Trial registration: Reference ISRCTN71172551 was assigned to this study.

\section{References}

1. Roland PS, Smith TL, Schwartz SR, et al. Clinical practice guideline: cerumen impaction. Otolaryngol Head Neck Surg. 2008;139(3)(Suppl 2):S1-S21.

2. Quality Improvement Scotland NHS. Best Practice Statement: Ear Care Edinburgh: Scottish Excecutive; 2006.

3. Coppin R, Wicke D, Mehta R, Little P. Management of earwax in primary care-postal survey of UK GPs and practice nurses. Fam Pract. 2004;21(4):413-414.

4. Clegg AJ, Loveman E, Gospodarevskaya E, et al. The safety and effectiveness of different methods of earwax removal: a systematic review and economic evaluation. Health Technol Assess. 2010;14(28):1-192.
5. Burton MJ, Doree C. Ear drops for the removal of ear wax. Cochrane Database System Rev. 2009,Jan 21;(1):CD004326.

6. American Academy of Otolaryngology. Head and Neck Surgery. Earwax. http://www.entnet.org/HealthInformation/earwax.cfm. Accessed Jun 29, 2010

7. Harris PG. A controlled trial of a ceruminolytic in general practice. Clin Trials J. 1968;5:1129-1133.

8. Coppin R, Wicke D, Little P. Managing earwax in primary care: efficacy of self-treatment using a bulb syringe. Br J Gen Pract. 2008; 58(546):44-49

9. Zhang J, Yu KF. What's the relative risk? A method of correcting the odds ratio in cohort studies of common outcomes. JAMA. 1998; 280(19):1690-1691.

10. Guest JF, Greener MJ, Robinson AC, Smith AF. Impacted cerumen: composition, production, epidemiology and management. QJM. 2004;97(8):477-488.

11. Grossan M. Cerumen removal-current challenges. Ear Nose Throat J. 1998;77(7):541-546, 548

12. Sharp JF, Wilson JA, Ross L, Barr-Hamilton RM. Ear wax removal: a survey of current practice. BMJ. 1990;301(6763):1251-1253.

13. Blake $P$, Matthews $R$, Hornibrook J. When not to syringe an ear. N Z Med J. 1998;111(1077):422-424.

13. Dinsdale RC, Roland PS, Manning SC, Meyerhoff WL. Catastrophic otologic injury from oral jet irrigation of the external auditory canal. Laryngoscope. 1991;101(1, pt 1):75-78. 